\title{
MODELLING AND EVALUATION OF A MULTI-AGENT SYSTEM FOR A COLLABORATION
}

\author{
Narjes Khezami, Samir Otmane and Malik Mallem \\ Complex System Lab, University of Evry \\ \{khezami, otmane, mallem\}@iup.univ-evry.fr
}

\begin{abstract}
We present a model of the MAS (Multi-Agent System) devoted to collaborative teleoperation architectures. The proposed MAS formalism is based on a new approach combining astutely two research area: MAS and CSCW (Computer Supported Collaborative Work). In the first area, a formal model of the MAS (Ferber approach) is used to give us features of the agent. The second area provides us a useful approach of collaboration based on the communication, coordination and production theories.

A formal model of the collaboration is proposed. This formalism is combined astutely with the Ferber model to provide new collaborative features of the agent. The result formalism is used to achieve the conceptual architecture of the MAS supporting full collaborative teleoperation on the Internet. Finally the system is evaluated and some results are discussed. Copyright (C) 2005 IFAC
\end{abstract}

Keywords: Agents, Computer-aided Work, Co-operation, Co-ordination, Group work, Modelling, Teleoperation.

\section{INTRODUCTION}

Several robot systems, which can be operated over the WWW, have been developed over the last few years (Hu, et al., 2001; Safaric, et al, 2001; Otmane, et al., 2000; Nasa, 2004). On the other hand, many current teleoperation architectures allow Human Operator (HO) to perform remote complex tasks and let multi-user interface for only collaborative control of the robot (Goldberg, et al., 2000; Goldberg, et al., 2002).

Here we consider the problem of the full collaborative teleoperation architecture. Questions are how to conceive and to develop adequate collaborative teleoperation architecture tacking a count of communication, coordination and production features? And how evaluate the developed collaborative systems?

In this paper we attempt to answer by proposing a new formalism of collaboration with a conceptual model useful to develop and evaluate the appropriate collaborative architecture.

Agents represent a fundamentally new way of considering complex distributed systems, containing societies of cooperating autonomous components. If we aim to build such systems, then principled techniques will be required for their design and implementation. We aim to assist the development of such systems by providing formalisms and notations that can be used to specify the desirable behaviour of agents and MAS; a requirement is that we should be able to move in a principled way from specifications of such systems to implementations. The features identified by using formalism serve to measure and evaluate implementations of agent systems.

In this paper we present a formal model of the MAS devoted to collaborative teleoperation architectures. The proposed MAS formalism is based on a new approach combining astutely two research area: MAS and CSCW (Laurillau, 2002; Ellis, et al., 1991; Salber, 1995). In the first area, a formal model of the MAS (Ferber approach) (Ferber, 1997) is used to give us features of the agent. The second area provides us a useful approach of collaboration based on the communication, coordination and production theories.

In the section 2, collaboration modelling is presented. The section 3 is devoted for the formal modelling for the collaborator agent. In section 4, the conceptual model for is given. In section 5, the implementation and the results evaluation are introduced. Some conclusions and futures works are presented in the section 6 . 


\section{COLLABORATION MODELLING}

\subsection{Collaboration specification}

The CSCW is the field of study that examines how technology affects group interaction, and how technology can best be designed and built to facilitate group work. Activities in that domain are known by the notions of groupware. Ellis (Ellis, et al., 1991) defines groupware as "computer-based systems that support groups of people engaged in a common task (or goal) and that provide an interface to a shared environment." While groupware refers to real computer-based systems, means the notion CSCW the study of tools and techniques of groupware as well as their psychological, social and organizational effects.

To structure groupware analysis and to propose a means of classification of the many tools permitting to reach the CSCW objectives, functionalities of the cooperative tools are divided in the functional clover as shown in Fig.1 (Laurillau, 2002; Salber, 1995). Then a groupware application covers three kinds of services: production, communication and coordination.

- The communication space refers to person-toperson communication such as e-mail, relay chat, mediaspace.

- The coordination space covers activities dependencies including temporal relationships between the multi-user activities. It also refers to the relationships between actors and activities.

- The production space refers to the objects produced by a group activity or to the objects shared by multiple users.

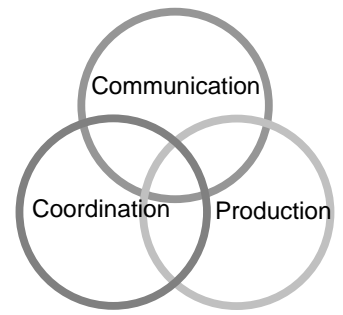

Fig. 1 Clover's Model

The importance of each of the three spaces depends on the nature of tools considered. So, the distinction according to the three spaces isn't strict because it's possible that a coordination activity takes place after a communication activity. For example, to make an appointment with someone on the phone is a coordination act based on a communication one.

Moreover, the global functional role can evolve in the course of time: this evolution can be modeled with the functional clover. For example, at the time of the production system use, it is possible, on a given moment; that the group activity will be centered on communication in order to coordinate, in spite of redefine the production activity.

\subsection{Collaboration formalism}

As shown in section 1, the collaboration is characterized by three spaces that are communication, coordination and production.

A collaboration system requires, at least, the existence of two agents interacting together with the aim of fulfilling jointly a task or overtaking jointly a particular goal. If an agent $i$ asks agent $j$ to collaborate with it (called Coll ${ }_{j}^{i}$ and represented by Eq. 1), he must firstly communicate with it by sending a request $\left(I n f_{i j}\right)$. The second agent sends a response to the first one $\left(\operatorname{Inf}_{j i}\right)$. If the response is positive, then they communicate together ${\text { ( } \mathrm{Comm}_{j}^{i}}^{i}$ Eq. 2) in order to coordinate; else the agent $i$ looks for other collaborator. The coordination is the result of the communication; if in the course of communication, agents reach an agreement to coordinate, then the coordination ( $\mathrm{Coor}_{i j}$ Eq. 3) can take place. During coordination, agents put on the actions plan that each one of them must performs (Actions $_{i}$ and Actions ${ }_{j}$.) Then, they execute their actions ( $\operatorname{Prod}_{i}$ and Prod ${ }_{j}$ Eq. 4) next they obtain partial results: Re sults $_{i}$ and Re sults ${ }_{j}$. Once partial results are obtained, those are combined (with the combination operator) in order to get the production global result. For more details see (Khezami, et al., 2004).

$$
\begin{aligned}
& \operatorname{Coll}_{j}^{i}=<\operatorname{Comm}_{j}^{i}, \operatorname{Coor}_{i j}, \operatorname{Prod}_{i}, \operatorname{Prod}_{j}> \\
& \operatorname{Comm}_{j}^{i}\left(\operatorname{Inf}_{i j}\right)=\left\{\left(\operatorname{Inf}_{i j}, \operatorname{Inf}_{j i}\right)\right\} \\
& \left.\operatorname{Coor}_{i j}\left(\left\{\operatorname{Inf}_{i j}, \operatorname{Inf}_{j i}\right\}\right)=\text { Actions }_{i}, \text { Actions }_{j}\right\} \\
& \operatorname{Prod}_{i}\left(\left\{\text { Actions }_{i}\right\}\right)=\left\{\text { Re sults }_{i}\right\}
\end{aligned}
$$

We consider the global collaboration (Eq. 5) between $N$ agents.

$$
\operatorname{coL}(\{i=1 \cdots N\})=\langle\operatorname{COMM}(\{i=1 \cdots N\}), \operatorname{COOR}(\{i=1 \cdots N\}), \operatorname{PROD}(\{i=1 \cdots N\})\rangle(5)
$$

The global communication is represented by all the couple of information exchanged agent-to-agent, for each agent which communicates with another (Eq. 6).

$$
\operatorname{COMM}(\{i=1 \cdots N\})=\left\{\left(\operatorname{Inf}_{i j}, \operatorname{Inf}_{j i}\right) / i=1 \cdots N-1, j=i+1 \cdots N\right\}(6)
$$

The global coordination of the system is represented by the set of all agents actions (Eq. 7).

$$
\operatorname{COOR}(\{i=1 \cdots N\})=\left\{\text { Actions }_{i} / i=1 \cdots N\right\}
$$

And the global production is the combination of partial results (Eq. 8).

$$
\operatorname{PROD}(\{i=1 \cdots N\})=\prod_{i=1 \cdots N}\left(\operatorname{Re} \text { sults }_{i}\right)
$$

\section{FORMAL MODELLING OF COLLABORATOR AGENT}

In this section we present a formal model for the collaborator agent. This model, as shown in Fig. 2, is the combination of the collaboration modeling 
presented in the previous section and the formal model of SMA presented in (Ferber, 1997) (Ferber's Model.)

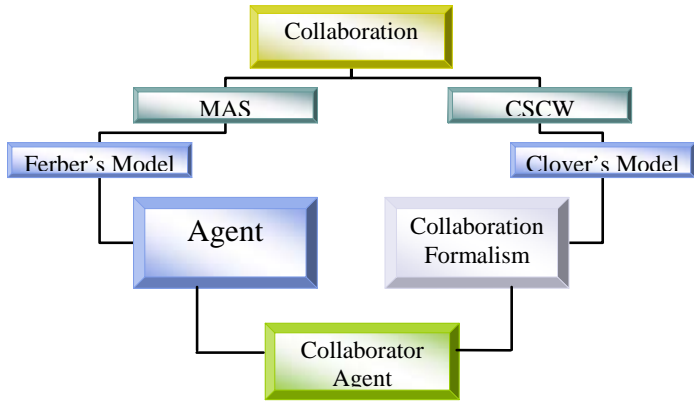

Fig. 2 Collaboration Modeling Process

A collaborator multi-agent system is a couple $\langle i, w\rangle$, where $i$ is a collaborator agent and $w$ is the world in which the agent evolve (Eq. 9).

$$
\begin{gathered}
i=\left\langle P_{i}, \text { Percept }_{i}, F_{i}, \text { Infl }_{i}\right\rangle \\
w=\langle\mathrm{E}, \Gamma, \Sigma, R\rangle
\end{gathered}
$$

Where,

- $\quad \mathrm{E}$ is the space in which the agent evolve. It's represented by agents in the environment.

- $\quad \Gamma$ is the set of agent actions, which modify the world evolution. For us, $\Gamma$ is the set formed by the three subsets $\left\{\operatorname{Inf}_{i j}\right\},\left\{\right.$ Actions $\left._{i}\right\},\left\{\right.$ Re sults $\left._{i}\right\}$ such as (Eq.10):

$$
\Gamma=\left\{\left\{\text { Inf }_{i j}\right\},\left\{\text { Actions }_{i}\right\},\left\{\text { Re sults }_{i}\right\}\right\}
$$

- $\quad \Sigma$ is the set of agent states. For us a collaborator agent $i$ has three states: communicate, coordinate or produce (comm,coor, prod). As presented in Eq. 11.

$$
\Sigma=\{\text { comm,coor }, \text { prod }\}
$$

- $\quad$ Percept $_{i}$ is a set of stimuli and sensations that the composed of three subsets $\left\{\right.$ Inf $\left._{j i}\right\},\left\{\right.$ Actions $\left._{j}\right\},\left\{\right.$ Re sults $\left.{ }_{j}\right\}$ such as (Eq. 12):

$$
\text { Percept }_{i}=\left\{\left\{\text { Inf }_{j i}\right\},\left\{\text { Actions }_{j}\right\},\left\{\text { Re sults }_{j}\right\}\right\}
$$

- $\quad P_{i}$ is an agent perception function (Eq.(13).)

$P_{i}:\left\{\right.$ comm $_{i}$, Coor $\left._{i}, \operatorname{prod}_{i}\right\} \rightarrow\left\{\left\{\right.\right.$ Inf $\left._{j i}\right\},\left\{\right.$ Actions $\left._{j}\right\},\left\{\right.$ Results $\left.\left._{j}\right\}\right\}(13)$

- $F_{i}$ is the agent behaviour function that determines the agent's state from its perceptions and its previous state (Eq.14).

$$
\begin{gathered}
F_{i}:\left\{\text { comm }_{i}, \text { Coor }_{i}, \operatorname{prod}_{i}\right\} \times\left\{\left\{\text { Inf }_{j i}\right\},\left\{\text { Actions }_{j}\right\},\left\{\text { Results }_{j}\right\}\right\} \\
\rightarrow\left\{\text { comm }_{i}, \text { Coor }_{i}, \operatorname{prod}_{i}\right\}
\end{gathered}
$$

- $\quad$ Infl $_{i}$ the agent action function, that modify the evolution of the world while producing influences (Eq.15)

$\operatorname{Infl}_{i}:\left\{\right.$ comm $_{i}$, coor $\left._{i}, \operatorname{prod}_{i}\right\} \rightarrow\left\{\left\{\right.\right.$ Inf $\left._{i j}\right\},\left\{\right.$ Actions $\left._{i}\right\},\left\{\right.$ Results $\left.\left._{i}\right\}\right\}(15)$

- $\quad R$ the law of evolution of the world (Eq.16)

$$
\begin{gathered}
R:\left\{\operatorname{comm}_{i}, \text { Coor }_{i}, \operatorname{prod}_{i}\right\} \times\left\{\left\{\operatorname{Inf}_{i j}\right\},\left\{\text { Actions }_{i}\right\},\left\{\text { Results }_{i}\right\}\right\} \\
\rightarrow\left\{\operatorname{comm}_{i}, \text { Coor }_{i}, \operatorname{prod}_{i}\right\}
\end{gathered}
$$

\section{CONCEPTUAL MODELING OF COLLABORATOR AGENT}

In this section we present the conceptual model of our collaborator multi-agent system.

Our conceptual architecture is composed of PAC* agents (Calvary, et al., 1997; Nigay, et al., 1997). In which, every collaborator agent is composed of three agents dedicated respectively to the communication, the coordination and the production (Fig. 3). These agents are bound to an agent ciment, that we call agent collaboration. This last one ensures the communication, on one hand, between the three dedicated agents and on the other hand, between the collaborator agent and the other collaborator agents. In this architecture model, a collaborator agent interact with the other collaborator agents of the system through the intermediary of its agent collaboration and its dedicated agents (communication, coordination, production) except that every one of them communicate always with the corresponding agent of other collaborator agents.

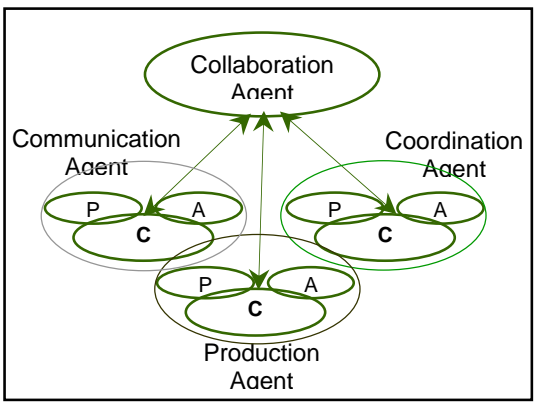

Fig. 3 Collaborator Agent

The collaboration agent ensures two principal functions; it allows communication between the three dedicated agents inside the collaborator agent; and it establishes a direct interaction between every dedicated agent and its corresponding one of other collaborator agents in order to communicate together without the help of agent collaboration. This direct communication between the three dedicated agents, inside a collaborator agent, and their corresponding agents of the other collaborator agents, allow to increase the efficiency of the system because the communication don't depend on the collaboration agent, so that avoid the "bottle neck" effect made by centralization.

On following, we present conceptual models of the three dedicated agents: communication, coordination and production (Fig. 4): 


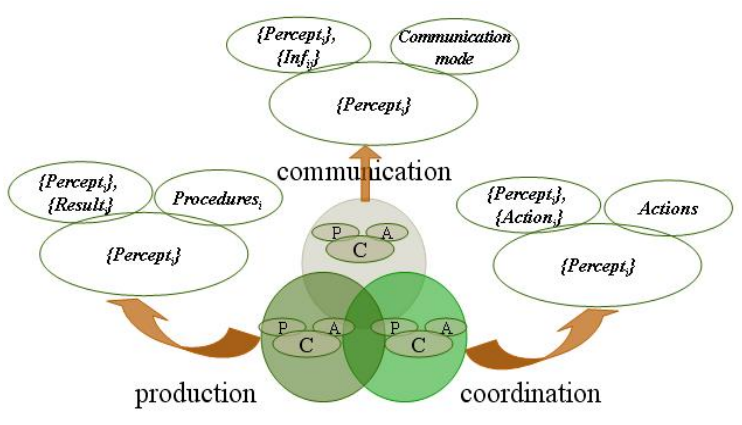

Fig. 4 Conceptual architecture of agent collaborator

\subsection{Agent communication}

The agent communication is the agent that decides if a mission can be executed or not, and therefore it is that who decides if collaboration can take place. Thus, the agent communication is the corner stone of the agent collaborator (knowing that an agent collaborator is composed of the four agents: communication, coordination, production and the agent collaboration that represent the agent ciment of the PAC* model). The agent communication defines the agent's state in functions of perceptions that it receives and its previous state. The agent communication has three facets.

- The control facet manages the information sent to the agent communication. This information presents different perceptions that the agent communication can receive from outside ( Percept $_{i}$.)

- The abstract facet is the facet that defines communication mode. The communication mode is defined according to the destination agent type (an external agent or an internal agent.)

- The presentation facet regroups the set of inputs and outputs of the agent communication and that are:

- $\quad\left\{I n f_{i j}\right\}$ are the set of information sent by the agent communication $i$ to the other ones $j$ (internal or external.)

- $\quad$ Percept $\left._{i}\right\}$ are the set of stimuli and sensations that the agent communication can receive.

\subsection{Agent coordination}

The agent coordination defines all the actions that the agent can accomplish. It is composed by three facets.

- The control facet manages perceptions that this agent can receive from outside ( Percept $_{i}$.)

- The abstraction facet defines all the actions that the agent collaboration can executes.

- The presentation facet regroups the set of inputs and outputs of the agent coordination: - Actions $\left._{i}\right\}$ are the set of executable actions.
- $\quad$ Percept $\left._{i}\right\}$ are the set of stimuli and sensations that this agent can receive.

\subsection{Agent production}

The agent production is the one responsible for executing actions stemming from agents collaboration. It composed from three facets:

- The control facet manages perceptions that this agent can receive from outside ( Percept $_{i}$.)

- The abstract facet regroups all necessary procedures for the execution of the different actions, defined by the agent coordination.

- The presentation facet defines the inputs and the outputs of the agent production :

- $\quad$ Percept $\left._{i}\right\}$ are the set of stimuli and sensations that the agent production can receive.

- $\quad\left\{\right.$ Re sults $\left._{i}\right\}$ are the set of results stemmed from the execution of actions.

\section{IMPLEMENTATION AND EVALUATION}

In this section the implementation and the evaluation of the collaboration system are presented.

\subsection{Implementation}

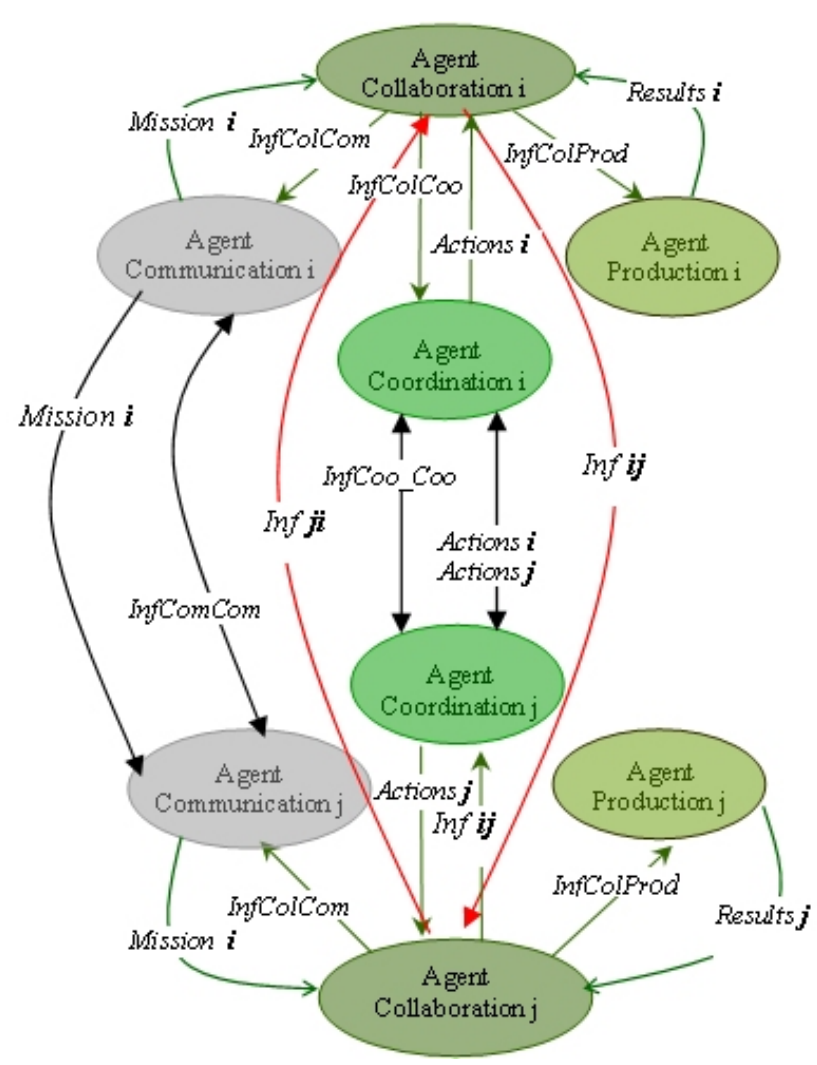

Fig. 5 Collaboration process between two agents

The collaboration model is implemented using the JADE framework (Java Agent DEvelopment 
framework) (Jade, 2004). JADE is a software development framework aimed at developing multiagent systems and applications conforming to FIPA standards for intelligent agents. JADE is written in Java language and is made of various Java packages. The Fig. 5 shows the collaboration process between two collaborator agents (agent collaborator $i$ and agent collaborator $j$.) The agent collaborator $i$ is the agent that starts collaboration process. All information between agents are represented by the Inf. Mission $_{i}$ is the mission chosen by the agent communication $i$ and the agent communication $j$.

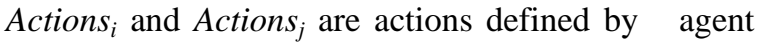
coordination $i$ and agent coordination $j$. Resluts $s_{i}$ and Results $_{j}$ are the confirmation of execution of the agents production $i$ and $j$.

\subsection{Evaluation of the collaboration model}

This section deals with evaluation of the multi-agent system for collaboration. The aim of this evaluation is to validate the collaboration model. Furthermore, evaluation is achieved by simulation agent's software execution.

The simulation took place on a portable Pentium 4, 512 Mo of RAM and 40 Go of hard drive, working under the Linux platform. The used programs are the JDK 1.4.2 and JADE 3.2.

For simulation, we made that every agent collaborator collaborate at least once. Then we varied the number of agent collaborator of the system of 2 until 200 for some experimentation and of 2 until 100 for other tests. For every variation we did 10 tests, after we took the average of every test.

The Fig. 6 shows the average rate of collaboration. The red curve shows the average collaboration rate by agent collaborator wished. That means, if we estimate that every agent collaborator collaborates at least once, we get the red curve that shows the average collaboration rate wished according to the number of agent collaborator of the system. The blue curve shows the average rate of real collaboration of every agent collaborator.

In order to estimate values of the collaboration rate wished, we used the uniform probability law whose samples are equiprobables (the collaboration probability of an agent with another such as the system contain $N$ agents is $1 / N$.) It comes back to consider that in a good system an agent must collaborate to the minimum with another agent.

Fig. 7 and Fig. 8 show the rate of information sent by every existing agent in the system according to number of collaborator agents. We have separated in two figures because the scale of the production information rate is too weak. The rate of information demonstrates an agent's importance in the collaboration process. An agent, that has a lot of interactions with the other agents, is an agent very implied in the collaboration and otherwise its average of information is raised more. The rate of information is calculated according to the quantity of messages sent by every agent, in other words which represents the communication rate of every agent with the other agents. This rate is calculated with (Eq. 17.) defining the message entropy.

$$
e(m)=-N\left(\frac{N_{0}}{N} \log \frac{N_{0}}{N}+\frac{N_{1}}{N} \log \frac{N_{1}}{N}\right)
$$

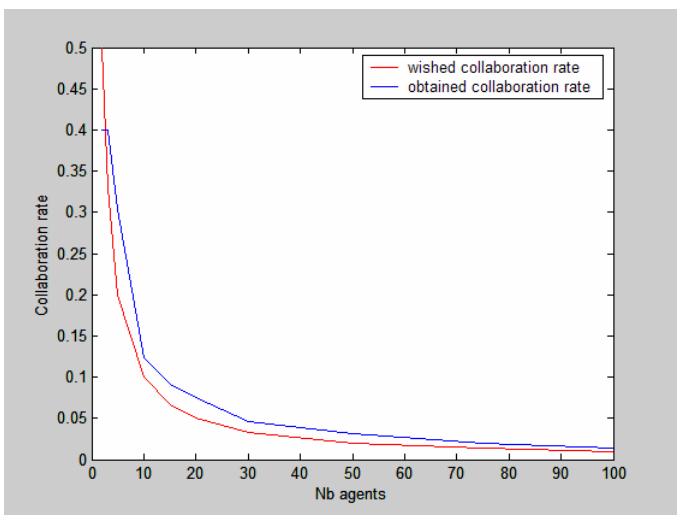

Fig. 6 The collaboration rate

We binarise the message and we calculate the message entropy $e(m)$, where $m$ is the message, $N_{0}$ is a number of zero of the message, $N_{1}$ is a number of one and $N$ is the sum of both.

Curves of the Fig. 7 show that: when the number of agents increases, it is the agent coordination that will have the biggest wage costs (because it negotiates all actions to execute and since the number of actions increases when the number of missions increases; and the number of missions increases when the collaboration becomes therefore more important the wage costs of the agent coordination increases.) The agent communication is the second because that is it that negotiates missions with the other agents. And the agent collaboration has less work because it makes only coordinate agents to communicate between them (section 5.1.)

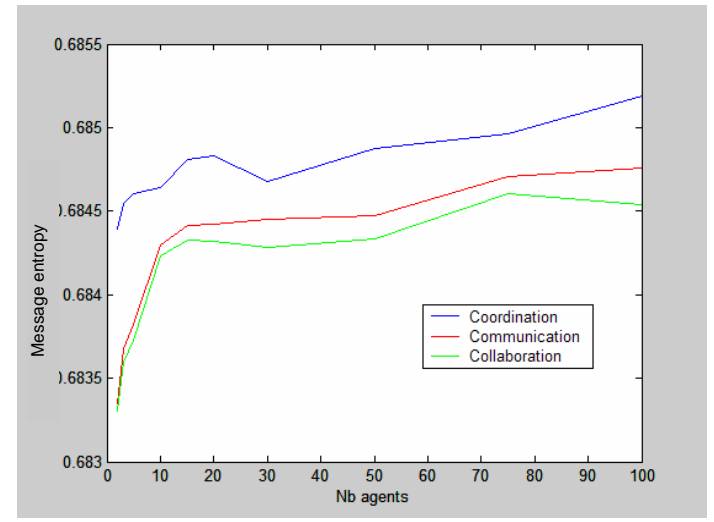

Fig. 7 Message entropy of communication, coordination and collaboration 
The curve of the Fig. 8 shows that the agent production is the less implied in steps of the collaboration because it executes orders transmitted by its agent collaboration and it has to send only confirmations when the execution is finished.

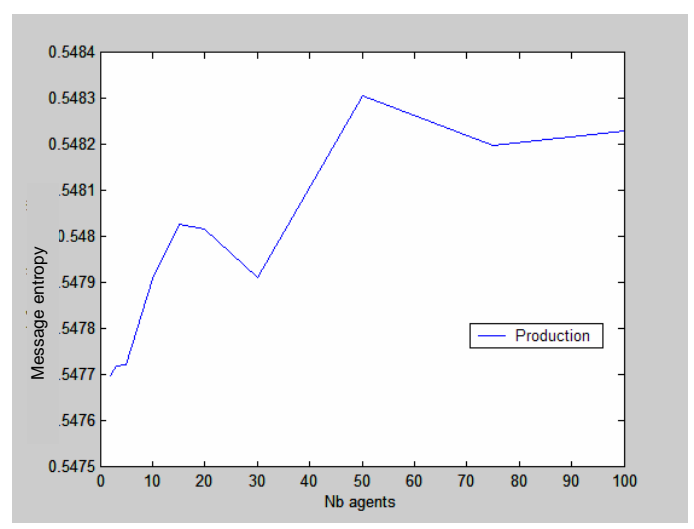

Fig. 8 Message entropy of production

Results show that the multi-agent system for the collaboration is not expensive in time of execution and that all implied agents make their work. We remark also that when the number of agent increases, the collaboration rate decreases; that mean if the number of agents increases, the probability that an agent collaborate decreases. It remains us to test it with a real system of collaboration and to see what that gives.

\section{CONCLUSION}

A model of the collaboration is proposed. This formalism is combined with the Ferber's model to provide new collaborative features of the agent. The result formalism is used to achieve the conceptual architecture of the MAS supporting full collaborative teleoperation on the Internet. The first application of this work is to use the proposed architecture to extend our experimental ARITI system (Otmane, et al., 2000; Otmane, et al., 2002; Nasa, 2004) with collaboration features. In fact ARITI system allows only one user to accomplish a task of teleoperation. It allows first connected to control a robot via Internet, this robot is located in our research laboratory. If a second user connects, he is only allowed to supervise the first one during his manipulation, or to prepare a teleoperation mission on simulation. Therefore the multi-agent system of collaboration is integrated in the kernel of ARITI in order to make it multi-user, i.e. allow several users to collaborate and control the robot at the same time. Consequently the system of collaboration manages the communication between users, conflicts of task execution as well as the different user behaviour.

\section{REFERENCES}

Calvary, G., Coutaz, J. and Nigay, L. "From SingleUser Architectural Design to PAC*: a Generic
Software Architecture Model for CSCW”. Actes de la conférence ACM Conference on Human Factors and Computing Systems (CHI'97), 1997, pages 242-249, ACM Press.

Ellis, Calernce, Gibbs, Simon, Rein and Gail «Groupware :some Issues and experiences ». Journal, communications of the ACM (CACM), 1991, volume 34, number 1, pages 38-58, ACM Press.

Ferber, J. “Les systèmes Multi-Agents: un aperçu général”, Techniques et sciences informatiques, Volume 16, 1997.

Goldberg, K., Chen, B., Solomon, R., Bui, S., Farzin, B., Heitler, J., Poon, D. and Smith, G. "Collaborative Teleoperation via the Internet". IEEE International Conference on Robotics and Automation San Francisco, Avril 2000.

Goldberg, K., Song, D., Khor, Y., Pescovitz, D., Levandowski, A. , Himmelstein, J., Shih, J., Ho, A., Paulos, E. and Donath. J." Collaborative Online Teleoperation with Spatial Dynamic Voting and a Human "Tele-Actor" ”, IEEE International Conference on Robotics and Automation, 14 mai 2002.

Hu, H., Yu, L., Tsui, P., and Zhou, Q. Internet-based robotic systems for teleoperation. Assembly Automation Journal, 21(2), 2001.

Jade web site, http://sharon.cselt.it/projects/jade/, 2004.

Khezami, N., Otmane, S., Mallem, M., and Ouramdane, N. "A "Formal Model for Collaborative Teleoperation”. The International Conference on Computing, Communications and Control Technologies (CCCT 2004), 14-17, Août 2004. Texas (USA).

Laurillau, Y. "Conception et réalisation logicielles pour les collecticiels centrées sur l'activité de groupe : le modèle et la plate-forme Clover". Phd. Thesis on Computer Science, University Joseph Fourier, Grenoble, France, September 2002.

Nasa space telerobotics program web site. http://ranier.oact.hq.nasa.gov/telerobotics page/, 2004.

Nigay, L. and Coutaz, J. "Software architecture modelling: Bridging Two Worlds using Ergonomics and Software Properties.” Livre, Formal Methods in Human-Computer Interaction (Palanque et Paterno éditeurs), 1997, pages 49-73, Springer Verlag.

Otmane, S., Khezami, N. and Mallem, M. "Distributed Predictive Display for Collaborative Teleoperation on the Internet”, in 4th Virtual Reality International Conference (VRIC2002), Pages 111-116, 19-21 june 2002, Laval (France).

Otmane, S., Mallem, M., Kheddar, A. and Chavand, F. "Ariti: an augmented reality interface for teleopeation on the internet". In Advanced Simulation Technologies Conference (ASTC2000) - High Performance Computing, pages 254-261, Wyndham City Center Hotel, Washington, D.C., USA, April 16-20 2000.

Safaric, R., Debevc, M., Parkin, R. and Uran, S. Telerobotics experiments via internet. IEEE Transactions on Industrial Electronics, 48(2):424-31, April 2001.

Salber, D. «De l'interaction individuelle aux systèmes multi-utilisateurs: L'exemple de la communication Homme-Homme Médiatisée ». Phd. Thesis on Computer Science, University Joseph Fourier, Grenoble, France, September 1995. 